\title{
Genetic testing in children and adolescents with intellectual disability
}

\author{
Nick Bass ${ }^{\mathrm{a}}$ and David Skuse ${ }^{\mathrm{b}}$
}

\begin{abstract}
Purpose of review
Investigation for genetic causes of intellectual disability has advanced rapidly in recent years. We review the assessment of copy number variants (CNVs) and the use of next-generation sequencing based assays to identify single nucleotide variation in intellectual disability. We discuss the diagnostic yields that can be expected with the different assays. There is high co-morbidity of intellectual disability and psychiatric disorders. We review the relationship between variants which are pathogenic for intellectual disability and the risk of child and adolescent onset psychiatric disorders.
\end{abstract}

\section{Recent findings}

The diagnostic yields from genome wide CNV analysis and whole exome sequence analysis are high - in the region of 15 and $40 \%$, respectively - but vary according to exact referral criteria. Many variants pathogenic for intellectual disability, notably certain recurrent $\mathrm{CNV}$ s, have emerged as strong risk factors for other neurodevelopmental disorders such as autism spectrum disorders, attention deficit hyperactivity disorder, and schizophrenia.

\section{Summary}

It is now conceivable that etiological variants could be identified in the majority of children presenting with intellectual disability using next-generation sequencing based assays. However, challenges remain in assessment of the pathogenicity of variants, reporting of incidental findings in children and determination of prognosis, particularly in relation to psychiatric disorders.

\section{Keywords}

chromosomal microarray analysis, developmental disorders, intellectual disability, whole exome sequencing, whole genome sequencing

\section{INTRODUCTION}

Half of all mental health problems encountered in adulthood have already been established by the age of 14 , and up to $75 \%$ by age 24 [1]. Ten percent of children aged 5-16 years have a diagnosable problem such as conduct disorder, anxiety disorder, attention deficit hyperactivity disorder (ADHD), or depression [2]. These figures are substantially higher in children with intellectual disability [3]. DSM-5 [4] defines intellectual disability as a disorder with onset during the developmental period that adversely affects both intellectual and adaptive functioning, causing deficits in conceptual, social, and practical domains. Mild, moderate, severe, and profound degrees of disability are defined on the basis of adaptive functioning nowadays, rather than in terms of IQ test results. This is because every day reasoning and judgment-making by people with intellectual disability is often poorer than formal cognitive assessments imply. In the United Kingdom, the management of people with intellectual disability is led by local specialist learning disability services, if these are available.

In most cases, the cause of intellectual disability is unknown, especially in people who have a nonsyndromic condition that lacks physical signs. Genomic variation is probably the leading cause of mild intellectual impairment in the general population. We each possess around 3 million polymorphic nucleotide variants in our genomes, the great majority of which are 'common' in the sense that we

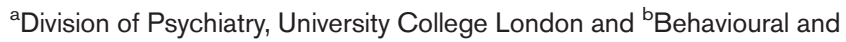
Brain Sciences Unit, Institute of Child Health, London, United Kingdom Correspondence to David Skuse, University College London, London, UK. Tel: 0207905 2168; e-mail: d.skuse@ucl.ac.uk

Curr Opin Psychiatry 2018, 31:490-495

DOI:10.1097/YCO.0000000000000456

This is an open access article distributed under the terms of the Creative Commons Attribution-Non Commercial License 4.0 (CCBY-NC), where it is permissible to download, share, remix, transform, and buildup the work provided it is properly cited. The work cannot be used commercially without permission from the journal. 


\section{KEY POINTS}

- CMA detects pathogenic variants in approximately $15 \%$ of children with developmental delay.

- WES may detect pathogenic variants in more $50 \%$ of children with developmental delay if used as a first tier diagnostic test and where parents are available for genotyping.

- Some variants pathogenic for intellectual disability, particularly CNVs, are strong risk factors for other neurodevelopmental disorders such as schizophrenia, ADHD and ASD.

- There is a paucity of information about specific associations of childhood onset psychiatric disorders with more recently identified variants pathogenic for intellectual disability.

- Systematic psychiatric phenotyping in genomic intellectual disability disorders is important to inform prognosis and facilitate early intervention.

share those variants with a substantial minority of other healthy individuals. These are often called single nucleotide polymorphisms (SNPs), defined as genomic differences in a single nucleotide, at a particular position in the genome where such variation is not rare. The cumulative impact of such variants, which occur in over $90 \%$ of all human genes, accounts for individual differences in complex physical and cognitive characteristics such as height and general intelligence. Most mild intellectual disability is probably attributable to common variation. Polygenic variation is also thought to contribute most genetic risk to the development of autism spectrum disorders (ASDs) [5].

A small proportion of such variants are unique, or almost unique to us as individuals (although they may also be found in our blood relatives). These are often known as private mutations, or as single nucleotide variants (SNVs). If they occur in a protein coding or regulatory part of a gene, they may alter genetic function if they are nonsynonymous with the typical nucleotide at that position. SNVs that arise in the germline of egg or sperm are termed 'de novo'. Disruptive SNVs are often associated with rare syndromes, and in a substantial proportion of such syndromes there is associated intellectual disability [6,7]. De-novo SNVs are thought to be responsible for most severe and profound intellectual disability [8], because affected individuals are unlikely to reproduce (and therefore do not pass on the mutation to future generations). SNVs in over 700 genes have been identified as contributing to autosomal dominant, autosomal recessive, and
X-linked intellectual disability [9]. Whole exome sequencing (WES) can detect de-novo coding mutations if they occur within genes, but many SNVs are in intergenic regions, hence are not picked up by this technique. Whole genome sequencing (WGS) has the capacity to identify rare variants in the regions between genes too and is reported to find coding mutations and potentially pathogenic structural changes in up to $60 \%$ of severe/profound intellectual disability cases. However, the interpretation of WGS-identified noncoding (intergenic) variants is problematic because we know relatively little about their impact on gene regulation [7].

Another class of genetic anomaly is responsible for many cases of intellectual disability. Copynumber variants (CNVs) are structural changes in the genome that may duplicate or delete a segment of DNA. Such CNVs may be inherited or they may arise de novo, and they are usually between $15 \mathrm{~kb}$ and $1 \mathrm{Mb}$ in length. They contribute to a range of neurodevelopmental disorders in both childhood [10] and adulthood [11"']. Not all CNVs are pathogenic [12], but pathogenic CNV are found in up to $15 \%$ of children referred for genetic investigation of developmental delay [13]. Some CNVs are particularly strongly associated with intellectual disability [14], especially the milder forms, and these are often familial. Recent research has shown, in both European and North American general population cohorts, that deletions of medium size and large duplications of DNA have a small but measurable detrimental impact on the IQ, and educational achievement, of people who possess them. The explanation is thought to be that large CNVs disrupt the action of many genes within the affected region, and the detriment to cognition is therefore polygenic in origin $\left[15,16^{\boldsymbol{*}}, 17^{\mathbf{*}}\right]$.

\section{GENETIC TESTING IN INTELLECTUAL DISABILITY}

Chromosomal microarray analysis (CMA) encompass all types of array-based genomic analyses, including array-based comparative genomic hybridization (aCGH) and SNP arrays. To identify potentially pathogenic CNVs, DNA testing with aCGH is often the first-line diagnostic test for children with intellectual disability, in both Europe and North America $\left[18,19^{-2}, 20\right]$. The introduction of array-based copy-number analysis has led to the identification of both inherited and de-novo microdeletions and duplications in up to $15 \%$ of cases [21]. It is important to be aware that the attribution of pathogenicity to a $\mathrm{CNV}$ identified by a microarray is by no means straightforward, and there is no universally agreed standard in the United Kingdom. Many CNVs are excessively rare events, and conclusions regarding 
the apparent strength of their association with cognition and psychiatric risk are therefore critically dependent on the interpretation of genomic data from unaffected comparison populations. In that regard, some variants previously considered to be pathogenic are being re-evaluated in light of increasing knowledge regarding their lack of association with disease in the general population.

WES is not routinely available in the UK's National Health Service, but some Regional Genetics Centres are using specialist panel arrays to detect small nucleotide variants that are known to be associated with intellectual disability and other disorders of neurodevelopment. The proportion of sporadic cases of intellectual disability caused by point mutations (SNV) is unknown. Exome sequencing has led to an increasing identification of de-novo variants [22], but in clinical practice, because of high locus heterogeneity, we often cannot with confidence attribute pathogenicity to individual mutations [8]. Evidence to guide such decision-making is slowly emerging from two recent UK national research studies that have recruited children with intellectual disability, of probable genetic etiology. The Deciphering Developmental Disorders (DDD) project employed genome-wide microarray and WES in a nationwide survey of children with complex developmental disorders of probable genetic origin [23"']. Damaging de-novo coding mutations were found in $42 \%$ of these previously investigated, yet undiagnosed, children, the vast majority ( $\sim 90 \%$ ) of whom had associated intellectual disability. The more recent 100000 Genomes project has used WGS to investigate a similar cohort, but the results are as yet unpublished [24].

\section{WHO GETS TESTED?}

A recent review, summarizing the outcome of several years of genetic testing for intellectual disability by a London community paediatric clinic [25"], stated referrals had been made by a wide range of paediatric specialists, general practitioners, therapists, and schools. Practitioners called for genetic testing when they predicted it was likely to be of diagnostic value, based on criteria that included significant developmental delay, an unusual physical phenotype, epilepsy and parental consanguinity. Rarely, if ever, was the reason for genetic testing in intellectual disability prompted by a neurodevelopmental disorder that manifested in terms of behavior. If a genetic diagnosis is made following CMA investigation, this may lead to counseling about the likely prognosis or potential complications associated with the disorder [ $\left.26^{*}\right]$. A positive genetic finding in association with intellectual disability can provide information about recurrence risk in any future children, following cascade testing of biological relatives. It is important to be aware that CMA testing is not without drawbacks. In addition to the challenges of determining the pathogenicity of many variants that could have contributed to the neurodevelopmental disability, there is the ethical dilemma of reporting incidental findings to the family of the affected child. Such incidental findings could include the discovery of risk variants for serious adult-onset diseases, such as breast cancer. Genetic testing of children with intellectual disability is nowadays focused on the preschool population, whereas most child psychiatry services do not routinely assess children under 6 years of age. However, a recent survey of child and intellectual disability psychiatrists in the United Kingdom found that just over half had directly ordered genetic investigations at some time. Although the majority of psychiatrists thought genetic diagnosis was helpful for the family, the responses suggested that the diagnosis did not often result in management changes [27"].

\section{GENETIC CAUSES OF ID AND RISK OF PSYCHIATRIC DISORDERS}

A broad range of childhood-onset psychiatric disorders is found in association with intellectual disability $[28,29]$, but there is rarely any evidence of a specific genetic cause, with the exception of some cases of ASD. In adults with intellectual disability, there is a better understanding of the risks of psychiatric comorbidity in those bearing some rare genetic anomalies $\left[30^{*}\right]$, such as a few well studied pathogenic CNVs, including schizophrenia's association with 22q11.2 or 1q21.1 microdeletions [31]. The reason for this paucity of knowledge is partly because the focus of previous research in children with intellectual disability has been on individuals with a small range of conditions, especially ASD [32"] and ADHD [33"], who are then subject to genetic screening. If damaging variants are found that are excessively rare in controls, there is a tendency to assume specificity. But because of high locus heterogeneity, it is hard to draw firm conclusions about the specific psychiatric pathogenicity of individual mutations [8]; mutations in genes associated with intellectual disability cause a wide range of phenotypes [34]. The alternative approach, undertaking broad psychiatric phenotyping of a representative sample of children with intellectual disability who have pathogenic genetic anomalies, is essential in order to set existing findings in context.

An excess of males is ascertained with neurodevelopmental disorders, including intellectual disability. The reasons for this bias is not known, but it is apparently not attributable to X-linked variants 
because 'monogenic' X-linked intellectual disability accounts for no more than $8 \%$ or so of male cases [35], and a wide range of epidemiological studies has shown that the excess of males over females is up to $50 \%$. This observation has been linked to the theory that for females, at genetic risk, to manifest the phenotype of neurodevelopmental dysfunction they need to possess higher mutational burden than males [36]. The phenomenon has been termed the female protective effect [37]. We know that males with normal-range IQ are more likely to be referred for genetic testing than females carrying the same autosomal variant, in populations with ASD. In the Simons Simplex Collection (SSC) of individuals with ASD, rare truncating SNVs show a slight female excess [38], but there are significantly more females than males with large (more than $400 \mathrm{~kb}$ ) CNVs. Where the CNV was familial, maternal transmission was significantly higher than paternal transmission for these large deleterious CNVs. This contrasts with evidence that the rate of de-novo point mutations is generally increased among older fathers [39].

\section{GENETIC TESTING IN INTELLECTUAL DISABILITY AND AUTISM SPECTRUM DISORDERS}

NICE guidelines in the United Kingdom [40] do not recommend routine genetic tests for children with an autistic disorder, but states these will be done 'as recommended by your regional genetics center, if there are specific dysmorphic features, congenital anomalies and/or evidence of a learning (intellectual) disability'. It used to be thought that children with autistic disorders were usually developmentally delayed. In a sense that is true, insofar as there is a substantially increased risk of ASD in children with intellectual disability [3], and consequently the apparent population prevalence of ASD is influenced by the prevalence of intellectual disability. In the United States, the 2014 National Health Interview Survey of Autism [41] used a revised question ordering and a new approach that asked about autistic characteristics before developmental disabilities. This change resulted in substantial increases in the apparent prevalence of autistic disorders because children formerly assigned as developmental disabilities were designated as having a primary diagnosis of ASD instead.

At a population level, most newly diagnosed autism is not nowadays associated with generalized learning disabilities, probably because the clinical ascertainment of autistic features in children of normal range intelligence has improved in recent years [42"]. The heritability of autism is very high [43"] implying shared, familial genetic risk factors increase the likelihood of the diagnosis. Most risk at a population level is because of common variation, but this acts additively with rare variation to enhance risk in those ASD cases who carry a strongly acting de-novo variant [44"]. A recent review of genetic risk in autism [45"] emphasized that CNVs that are associated with a high risk of autism overlap with those known to cause intellectual disability. Not only are they expressed in the brain but are especially likely to involve genes that are structurally or functionally engaged in chromatin remodeling and transcription regulation. CNVs that are particularly strongly associated with ASD include duplications of $16 \mathrm{p} 11.2$, deletions of $15 \mathrm{q} 13.3,2 \mathrm{p} 16.3$, and $15 q 11.2$ [46]. The yield of genetic testing of nonsyndromal cases of autism in simplex families (which are less likely than multiplex families to carry heritable private mutations) can be estimated from internationally curated samples. Large structural abnormalities (CNVs), which are detectable by microarray, can be found in up to $10 \%$ of cases [32"]. These CNVs are usually associated with relatively mild learning disabilities, and they comprise both inherited and de-novo anomalies. The wider use of exome sequencing is likely to increase the proportion of cases with an identifiable point mutation or indels that are the cause of loss of function or otherwise disrupting, the great majority of which are de novo (and likely to be paternal in origin [47]).

\section{CONCLUSION AND FUTURE DIRECTIONS}

In summary, when applied as a first-tier test for broadly defined developmental delay, current widely-available arrays (aCGH) detect pathogenic variants in approximately $15 \%$ of children. We know that, in a research context, WES gives a greater diagnostic yield of around $40 \%$ in children with severe developmental delay and it is estimated that yield could exceed $50 \%$ if used as a first tier diagnostic test [48"']. We anticipate that WGS may provide even better identification of pathogenic variants, although there is still debate about the interpretation of intergenic SNV. However, accurate assignment of pathogenicity to SNV is getting better, prompting some to advocate re-analyzing existing data sets $\left[48^{-"}, 49^{-1}\right.$. Economic analysis suggests that current use of WES reduces healthcare costs when applied to the investigation of intellectual disability $\left[50^{\circ}\right]$.

The falling cost of WGS, and the associated improvement in our ability to detect very small CNVs, makes it likely that the first-tier investigation of childhood intellectual disability will be WGSbased in the future. However, our understanding of the relationship between genotype and phenotype in intellectual disability and related 
neurodevelopmental disorders is at an early stage for the majority of variants. Epigenetic changes are also likely to have important modifying effect on these neurodevelopmental trajectories, but measurement of epigenetic changes and integration of this information and polygenic risk for prognostic predication represents a significant research challenge.

\section{Acknowledgements}

We thank Jeanne Wolstencroft, Hayley Denyer, and Nasri Fatih for their review of drafts of the study.

\section{Financial support and sponsorship}

MRC (UK) and MRF (UK) grant support. MR/L011166/1 and MR/NO22572/1. Grant funding: D.H.S., Principal Investigator. Intellectual Disability and Mental Health: Assessing Genomic Impact on Neurodevelopment (IMAGINE).

\section{Conflicts of interest}

There are no conflicts of interest.

\section{REFERENCES AND RECOMIMENDED}

\section{READING}

Papers of particular interest, published within the annual period of review, have been highlighted as:

- of special interest

m. of outstanding interest

1. Kessler RC, Berglund $P$, Demler $O$, et al. Lifetime prevalence and age-ofonset distributions of DSM-IV disorders in the National Comorbidity Survey Replication. Arch Gen Psychiatry 2005; 62:593-602.

2. Green $\mathrm{H}$, McGinnity Á, Meltzer $\mathrm{H}$, et al. Mental health of children and young people in Great Britain, 2004. Office for National Statistics.

3. Einfeld SL, Ellis LA, Emerson E. Comorbidity of intellectual disability and mental disorder in children and adolescents: a systematic review. J Intellect Dev Disabil 2011; 36:137-143.

4. DSM-5 American Psychiatric Association. Diagnostic and statistical manual of mental disorders. Arlington: American Psychiatric Publishing; 2013.

5. Gaugler T, Klei L, Sanders SJ, et al. Most genetic risk for autism resides with common variation. Nat Genet 2014; 46:881.

6. Wright CF, Fitzgerald TW, Jones WD, et al. Genetic diagnosis of developmental disorders in the DDD study: a scalable analysis of genome-wide research data. Lancet 2015; 385:1305-1314.

7. Gilissen C, Hehir-Kwa JY, Thung DT, et al. Genome sequencing identifies major causes of severe intellectual disability. Nature 2014; 511:344.

8. Rauch A, Wieczorek D, Graf E, et al. Range of genetic mutations associated with severe nonsyndromic sporadic intellectual disability: an exome sequencing study. Lancet 2012; 380:1674-1682.

9. Vissers LE, Gilissen C, Veltman JA. Genetic studies in intellectual disability and related disorders. Nat Rev Genet 2016; 17:9-18.

10. Thapar A, Cooper M. Copy number variation: what is it and what has it told us about child psychiatric disorders? J Am Acad Child Adolesc Psychiatry 2013; 52:772-774.

11. Marshall CR, Howrigan DP, Merico D, et al. Contribution of copy number

-1. variants to schizophrenia from a genome-wide study of 41,321 subjects. Nat Genet 2017; 49:27-35.

Large-scale association analysis of CNVs in schizophrenia utilizing over $20 \mathrm{~K}$ cases and $20 \mathrm{~K}$ controls. Although the samples came from different cohorts a centralized analysis platform was used. Genome wide significant evidence of schizophrenia risk was obtained for recurrent eight loci. A globally higher burden of CNVs was observed in schizophrenia cases compared to controls with enrichment in genes associated with synaptic function

12. Hehir-Kwa JY, Pfundt R, Veltman JA, de Leeuw N. Pathogenic or not? Assessing the clinical relevance of copy number variants. Clin Genet 2013; 84:415-421.

13. Miller DT, Adam MP, Aradhya S, et al. Consensus statement: chromosomal microarray is a first-tier clinical diagnostic test for individuals with developmental disabilities or congenital anomalies. Am J Hum Genet 2010; $86: 749-764$.
14. Coe BP, Witherspoon $K$, Rosenfeld JA, et al. Refining analyses of copy number variation identifies specific genes associated with developmental delay. Nat Genet 2014; 46:1063-1071.

15. Männik K, Mägi $R$, Macé $A$, et al. Copy number variations and cognitive phenotypes in unselected populations. JAMA 2015; 313:2044-2054.

16. Huguet $\mathrm{G}$, Schramm $\mathrm{C}$, Douard $\mathrm{E}$, et al. Measuring and estimating the effect

- sizes of copy number variants on general intelligence in community-based samples. JAMA Psychiatry 2018; 75:447-457.

The relationship between CNVs $50 \mathrm{~Kb}$ or larger and IQ was evaluated two general population cohorts (IMAGEN project and Saguenay Youth Study) using linear regressions. CNV size, number of genes/exons affected was inversely related to IQ with each deleted gene is associated with a mean (SE) decrease in PIQ of 0.67. However, this relationship was only observed for rare deletions.

17. Kendall KM, Rees E, Escott-Price V, et al. Cognitive performance among

- carriers of pathogenic copy number variants: analysis of 152,000 UK biobank subjects. Biol Psychiatry 2017; 82:103-110.

The relationship between 12 schizophrenia associated CNVs and 41 neurodevelopmental associated CNVs and results on seven cognition tests was examined in the UKBiobank data (first 152,728 genotyped individuals). Carriers of these $\mathrm{CNVs}$ were found to have significant impairments in cognitive performance.

18. Stankiewicz $P$, Beaudet $A L$. Use of array $C G H$ in the evaluation of dysmorphology, malformations, developmental delay, and idiopathic mental retardation. Curr Opin Genet Dev 2007; 17:182-192.

19. Hart AR, Sharma $R$, Atherton $M$, et al. Aetiological investigations in early devel- opmental impairment: are they worth it? Arch Dis Child 2017; 102:1004-1013. Retrospective casenote analysis of the diagnostic yield of 'screening' investigations for early developmental impairment in 699 participants referred to a tertiary service in the United Kingdom. MRI and microarray found to be highest yielding tests by large margin.

20. Moeschler JB, Shevell M. Comprehensive evaluation of the child with intellectual disability or global developmental delays. Pediatrics 2014; 134:e903-e918.

21. Cooper GM, Coe BP, Girirajan $S$, et al. A copy number variation morbidity map of developmental delay. Nat Genet 2011; 43:838-846.

22. De Ligt J, Willemsen $\mathrm{MH}$, Van Bon BW, et al. Diagnostic exome sequencing in persons with severe intellectual disability. New Engl J Med 2012; 367:1921-1929.

23. The Deciphering Developmental Disorders Study. Prevalence and architecture -1 of de novo mutations in developmental disorders. Nature 2017;542:433-438. Participants with severe undiagnosed developmental disorders were recruited from UK clinical genetics services. Exome sequencing was performed on 4293 families. Damaging de-novo mutations (DNM) were found to be enriched in 93 genes, including 14 genes that had not previously been strongly implicated in the etiology of intellectual disability. It was estimated that $42 \%$ of the cohort carried DNMs in coding sequence.

24. Torjesen I. Genomes of 100000 people will be sequenced to create an open access research resource. BMJ 2013; 347:f6690.

25. Best S, Rosser E, Bajaj M. Fifteen years of genetic testing from a London - developmental clinic. Arch Dis Child 2017; 102:1014-1018.

Retrospective case note analysis of all new referrals to Child Development Team (CDT) clinic in Tower Hamlets, East London, UK, between 1999 and 2013. 53.9\% had undergone genetic testing and $39.1 \%$ of those tested had a confirmed genetic diagnosis

26. Redley M, Pannebakker M, Holland A. Improving the health and well being of - adults with conditions of a genetic origin: views from professionals, syndrome support groups and parents. J Appl Res Intellect Disabil 2018; 31:62-75. Views on the utility of etiological genetic diagnoses for people with neurodevelopmental disorder were solicited from professional and members of syndrome support organizations. Support was found for a more medicalized approach to neurodevelopmental disorders with the aim of improving the health and wellbeing of people with intellectual disability

27. Wolfe K, Stueber K, McQuillin A, et al. Genetic testing in intellectual disability - psychiatry: opinions and practices of UK child and intellectual disability psychiatrists. J Appl Res Intellect Disabil 2018; 31:273-284.

Survey of attitudes towards and use of genetic investigations by child and adolescents psychiatrists and intellectual disability psychiatrists conducted through the UK Royal College of Psychiatrists.

28. Emerson E. Prevalence of psychiatric disorders in children and adolescents with and without intellectual disability. J Intellect Disabil Res 2003; 47:51-58.

29. Emerson E, Hatton C. Mental health of children and adolescents with intellectual disabilities in Britain. Br J Psychiatry 2007; 191:493-499.

30. Wolfe K, Strydom A, Morrogh D, et al. Chromosomal microarray testing in

- adults with intellectual disability presenting with comorbid psychiatric disorders. Eur J Hum Genet 2017; 25:66-72.

CMA of 202 adults with idiopathic ID and comorbid psychiatric disorders recruited from UK specialist ID services. A diagnostic yield of $11 \%$ was obtained with recurrent CNVs in the $15 q 11-q 13$ and $16 p 11.2-p 13.11$ regions being most frequently observed.

31. Baker K, Costain G, Fung WL, Bassett AS. Chromosomal microarray analysis-a routine clinical genetic test for patients with schizophrenia. Lancet Psychiatry 2014; 1:329-331.

32. Ramaswami G, Geschwind $\mathrm{DH}$. Genetics of autism spectrum disorder. - Handb Clin Neurol 2018; 147:321-329.

Recent review of the identification of ASD disease genes 
33. Thapar A. Intellectual disability and attention-deficit/hyperactivity disorder:

what does the clinical and genetic overlap mean for practice and research? J Am Acad Child Adolesc Psychiatry 2017; 56:105-106.

Editorial discussing recognition of ID in context of ADHD presentation in response to paper by Faraone et al. (The familial co-aggregation of attention-deficit/hyperactivity disorder and intellectual disability: a register-based family study. J Am Acad Child Adolesc Psychiatry 2017;56:167-174.)

34. Girirajan S, Rosenfeld JA, Coe BP, et al. Phenotypic heterogeneity of genomic disorders and rare copy-number variants. New Engl J Med 2012; 367:1321-1331

35. Chelly J, Mandel JL. Monogenic causes of X-linked mental retardation. Nat Rev Genet $2001 ; 2: 669-680$.

36. Jacquemont $S$, Coe $B P$, Hersch $M$, et al. A higher mutational burden in females supports a 'female protective model' in neurodevelopmental disorders. Am J Hum Genet 2014; 94:415-425.

37. Robinson $E B$, Lichtenstein $\mathrm{P}$, Anckarsäter $\mathrm{H}$, et al. Examining and interpreting the female protective effect against autistic behavior. Proc Natl Acad Sci 2013; 110:5258-5262.

38. Sanders SJ, He X, Willsey AJ, et al. Insights into autism spectrum disorder genomic architecture and biology from 71 risk loci. Neuron 2015; 87:1215-1233.

39. Hehir-Kwa JY, Rodríguez-Santiago $B$, Vissers LE, et al. De novo copy number variants associated with intellectual disability have a paternal origin and age bias. J Med Genet 2011; 48:776-778.

40. Kendall $T$, Megnin-Viggars $\mathrm{O}$, Gould $\mathrm{N}$, et al. Management of autism in children and young people: summary of NICE and SCIE guidance. BMJ 2013; 347:f4865.

41. Zablotsky B, Black LI, Maenner MJ, et al. Estimated prevalence of autism and other developmental disabilities following questionnaire changes in the 2014 National Health Interview Survey. 2015. National Health Statistics Reports, 87. November 13, 2015. U.S. Department of Health and Human Services, CDC.

42. Fombonne E. The rising prevalence of autism. J Child Psychol Psychiatry - 2018; 59:717-720.

Editorial charting the development of surveying methods and their relationship to the rise in prevalence estimates for ASD. The implications of the lack of a standardized methodology are discussed

43. Woodbury-Smith M, Scherer SW. Progress in the genetics of autism specn trum disorder. Dev Med Child Neurol 2018; 60:445-5139.

Recent review of our understanding of the genetic architecture of ASD, considering both contributions of rare and common variants to etiology. Discussion of the clinical implications of the advances in the genetics of ASD
44. Weiner DJ, Wigdor EM, Ripke S, et al. Polygenic transmission disequilibrium - confirms that common and rare variation act additively to create risk for autism spectrum disorders. Nat Genet 2017; 49:978-985.

Report of a novel approach - the polygenic transmission disequilibrium test - to examine polygenic risk in ASD. Polygenic risk for ASD, Schizophrenia, and higher educational attainment was found to be over transmitted to children with ASD. Furthermore, polygenic risk for ASD and rare ASD risk variants were found to act in an additive manner.

45. Muhle RA, Reed HE, Stratigos KA, Veenstra-VanderWeele J. The emerging - clinical neuroscience of autism spectrum disorder: a review. JAMA psychiatry 2018; 75:514-523.

Review of how ASD risk-related biological pathways are emerging through convergent lines of investigation (genetic, molecular biology and neuroimaging). Discussion of the potential for personalized medicine in ASD.

46. Levy $\mathrm{D}$, Ronemus $\mathrm{M}$, Yamrom $\mathrm{B}$, et al. Rare de novo and transmitted copynumber variation in autistic spectrum disorders. Neuron 2011; 70:886-897.

47. Kong A, Frigge ML, Masson G, et al. Rate of de novo mutations and the importance of father's age to disease risk. Nature 2012; 488:471-475.

48. Wright CF, McRae JF, Clayton S, et al. Genet Med 2018. doi: 10.1038/ gim.2017.246. [Epub ahead of print]

Re-analysis of WES data from the DDDs study led to an increase in the diagnostic yield from $27 \%$ in 2014 to $40 \%$. This study highlights the importance of re-analysis of sequence data while the rate of new pathological variant discovery remains high. The authors also estimate that using WES as a first tier test for developmental disorder in a trio paradigm would give a diagnostic yield in excess of $50 \%$

49. Nambot $S$, Thevenon J, Kuentz $P$, et al. Clinical whole-exome sequencing for

- the diagnosis of rare disorders with congenital anomalies and/or intellectual disability: substantial interest of prospective annual reanalysis. Genet Med 2018; 20:645-654.

Annual re-analysis of WES data from the investigation of children with congenital abnormalities/intellectual disability was undertaken. A diagnosis was made in $27.9 \%$ of patients for whom no diagnosis was made initially. International data sharing of translational research was successfully employed in addition to usual diagnostic procedures to assign variant pathogenicity.

50. Vrijenhoek T, Middelburg EM, Monroe GR, et al. Whole-exome sequencing in - intellectual disability; cost before and after a diagnosis. Eur J Hum Genet 2018. [Epub ahead of print]

Retrospective case note analysis of 370 patients who had undergone WES for investigation of intellectual disability. Pre-WES and post-WES costs were calculated for each patient. A large reduction in costs was found post-WES regardless of diagnostic outcome of WES. The authors advocate utilization of WES early in the diagnostic trajectory to maximize benefit. 